\title{
El caso de Paola Guzmán Albarracín, violencia sexual infantil en el ámbito educativo en Ecuador. ${ }^{1}$
}

The case of Paola Guzman Albarracin, child sexual violence in the educational field in Ecuador.

\author{
Cecilia Palomo Caudillo \\ Catedrática poder judicial \\ federal en Méjico \\ ceciliapalomoc@gmail.com
}

\section{Resumen}

El objetivo de este trabajo es reflexionar sobre la importancia de la educación para las niñas y mujeres en América Latina y el Caribe, a través del análisis de la primera sentencia de la Corte Interamericana de Derechos Humanos, relativa a hechos de violencia sexual en el ámbito educativo en los que se atribuye responsabilidad internacional a Ecuador. Se trata de Paola, una niña víctima de violencia sexual en el colegio, se abordan los principales hechos y argumentos, para evidenciar la necesidad de impulsar la formación en igualdad real desde todas las trincheras posibles, sobre todo en el escenario de pandemia que atravesamos.

Palabras clave: Violencia sexual, Educación, Niñas, Latinoamérica, Igualdad real.

\begin{abstract}
The objective of this work is to reflect on the importance of education for girls and women in Latin America and the Caribbean, through the analysis of the first judgment of the InterAmerican Court of Human Rights, regarding acts of sexual violence in the educational field, in which international responsibility is attributed to Ecuador. It's about Paola, a girl victim of sexual violence at school, the main facts and arguments are addressed, to show the need to promote education in real equality from all possible trenches, especially in the pandemic scenario that we are going through.
\end{abstract}

${ }^{1}$ Recibido: 17/02/2021 Evaluado: 03/03/2021 Aceptado: 23/04/2021 
Keywords: Sexual violence, Education, girls, Latin America, Real equality.

\section{Introducción}

De acuerdo con datos de UNICEF (2018), 72 millones de niñas y niños de 0-14 años viven en pobreza en América Latina, las comunidades rurales, indígenas y afrodescendientes sufren la pobreza de manera agudizada. Un panorama de desigualdad como este, unido a la cultura machista que prevalece en la región, provocan que 4 de cada 10 niñas entre 15 y 19 años experimenten violencia de género, 1.1 millón de niñas adolescentes han experimentado además violencia sexual.

La pobreza y la violencia dejan poco espacio para pensar en la educación. Según la OCDE (2018), 14 millones de niñas y niños están fuera del sistema educativo en América Latina y el Caribe. Esto arroja porcentajes casi cuatro veces más altos que en los países desarrollados. Esta exclusión educativa afecta especialmente a las niñas y adolescentes. De acuerdo al Banco Mundial (2018), menos de dos tercios de las niñas $(65,0 \%)$ completan su educación primaria, y solo una de cada tres $(34,4 \%)$ completa la escuela secundaria inferior.

Aunado a lo anterior, las pocas niñas que logran acceder a la educación, lo hacen en entornos escolares en los que, con frecuencia, se reproducen diversos tipos de violencias, en especial la que se dirige a ellas en razón de su género. Hablamos de la violencia sexual en el ámbito educativo, la misma que les causa sufrimiento físico, sexual y psicológico; llevándoles en casos extremos al suicidio. Según la UNICEF (2014), esta modalidad de violencia es perpetrada por los diferentes actores insertos en el ámbito educativo, y está basada en la discriminación, las relaciones de poder desiguales entre mujeres y hombres, los estereotipos de género y patrones culturales basados en el control, dominio, sumisión y devaluación de las mujeres y niñas.

Las investigaciones de CONTRERAS J.M. (2010), han asociado la violencia sexual con normas sociales como, culpar a las mujeres cuando son violadas o sufren otros tipos de violencia sexual; justificar la violencia perpetrada por hombres debido a sus "inherentes deseos sexuales"; ver a las mujeres y niñas como objetos sexuales; y el "culto a la virginidad de la mujer". En este plano, la violencia sexual se asocia también con una aceptación social más generalizada del uso de la violencia.

Este es el caso de Paola, una niña que ingresó a un colegio público para niñas en Ecuador a los 12 años, y desde los 14 fue abusada por el vicerrector de la escuela, se trata de un hombre de casi 60 años de edad, que contó con el silencio de profesoras, médicos, padres y madres de familia, así como autoridades estatales, para abusar no solamente de Paola, sino también de otras niñas del colegio. Tras dos años de abuso sexual y psicológico, Paola que se sentía "enamorada" de su explotador, decidió ingerir fósforo blanco para acabar con su vida. Su madre Petita es quien se encargó de luchar 18 años para llegar a la Corte Interamericana de Derechos Humanos (2020), se trata del primer caso de violencia sexual en el ámbito escolar resuelto por este tribunal regional de derechos humanos.

A continuación se sintetizan los hechos, así como los principales argumentos de la sentencia en lenguaje simple, con la intención de generar una reflexión sobre el largo camino que nos 
queda por recorrer hacia una igualdad sustantiva en la educación para las niñas y mujeres de América Latina y el Caribe.

\section{Los hechos}

\section{Contexto. Situación de violencia sexual en instituciones educativas en Ecuador.}

El contexto en el que Paola fue víctima de violencia en el colegio no constituyó un hecho aislado, en esa época existían ya varios informes de entidades internacionales y nacionales que se refirieron a una situación de violencia, acoso y abuso sexual en las instituciones educativas de Ecuador. Por mencionar algunas de ellas, en 1998 el Comité de los Derechos del Niño expresó su preocupación por la práctica del maltrato infantil en Ecuador, inclusive en la escuela; en aquel entonces recomendó establecer mecanismos adecuados para atender las denuncias sobre maltrato de niñas o niños. También expresó su preocupación por la incidencia de suicidios de muchachas y la insuficiencia del acceso por parte de los adolescentes a educación sobre salud reproductiva. De acuerdo con ese mismo Comité y otros del sistema de la Organización de las Naciones Unidas, dichas situaciones continuaron presentándose en períodos más recientes (2008, 2010, 2015 y 2017).

A su vez, la Organización Mundial de la Salud realizó un estudio en el que encontró que años antes de los hechos, en 1991, tres de cada diez niños y niñas encuestadas habían sufrido abuso sexual entre los 11 y los 16 años de edad; y que, en 2008, el $23.3 \%$ de las niñas y niños en Guayaquil reportaban haber sido víctimas de algún tipo de abuso sexual y que estas cifras tendrían una tendencia a aumentar si no se actuaba al respecto.

Por otra parte, información generada en el ámbito estatal de Ecuador durante el 2001, muestra que el abuso y el acoso sexuales eran problemas conocidos en el ámbito educativo, que no habían sido abordados en forma sistemática, ni se habían emprendido acciones sostenidas para su prevención, denuncia y sanción. En esa oportunidad, el Consejo Nacional de las Mujeres (CONAMU) concluyó que el acoso y el abuso sexual eran una realidad en el espacio educativo, y sostuvo que los profesores eran agresores típicos.

La historia de Paola sucede, por lo tanto, en un espacio educativo público que no solo carecía de medidas de prevención ante actos de violencia sexual, sino que también normalizaba tales conductas, las mismas que, en el caso de Paola, se produjeron de forma sostenida por un período prolongado de tiempo.

\section{La violencia sexual sufrida por Paola Guzmán Albarracín y su posterior suicidio.}

Paola del Rosario Guzmán Albarracín nació el 10 de diciembre de 1986 en Guayaquil. Era hija de Petita Albarracín y Máximo Guzmán. A partir de los 12 años asistió al Colegio Fiscal Técnico de Comercio y Administración Dr. Miguel Martínez Serrano, un establecimiento de educación pública para niñas, dependiente del Ministerio de Educación de la República del Ecuador.

Paola vivía en ese entonces con su madre, su abuela y su hermana menor. Según testimonios, en el año 2001, cuando tenía 14 años y cursaba el segundo año de educación básica, Paola 
comenzó a tener problemas con ciertas materias y el Vicerrector del colegio, Bolívar Eduardo Espín Zurtía, ofreció pasarla de año, con la condición de que mantuviera relaciones sexuales con él.

En una audiencia pública, la señora Petita mencionó que notó un cambio en Paola más o menos en octubre de 2001. Una prima de Paola declaró que Paola les informó que le faltaban puntos para pasar de año, pero que ella vería cómo arreglar ese asunto, que no se preocuparan, porque tenía un padrino dentro del colegio. También señaló que acompañó a la señora Petita a hablar con el vicerrector Bolívar Espín y que, al llegar Paola, él la tomó del hombro y le dijo: "pero yo ya hablé contigo, verdad princesita". Agregó, finalmente, que Paola le contó que el Vicerrector siempre la trataba así, "cariñosamente".

Los testimonios y declaraciones señalan que el personal del colegio conocía la relación entre ambos, y que Paola no había sido la única estudiante con la que el Vicerrector había tenido acercamientos de índole sexual, aunque estos hechos nunca fueron denunciados. Por el contrario, sí hubo acciones del personal del colegio tendentes a proteger al Vicerrector luego de la muerte de Paola.

El 11 de diciembre de 2002, la Inspectora del curso de Paola le envió una citación a su madre para que se presentara en el colegio al día siguiente. Según la Inspectora, dicha citación se debió a que una semana antes Paola había faltado a clases, y porque con frecuencia encontraba a la niña en el bar (cafetería) o en el patio en horas de clase, sin permiso.

El jueves 12 de diciembre de 2002, el mismo día de la cita que tendría su madre con la Inspectora, dos días después de cumplir 16 años, estando en su casa, entre las 10:30 hs. y las 11:00 hs. Paola ingirió unas pastillas, denominadas coloquialmente "diablillos", que contienen fósforo blanco. Luego se dirigió al colegio.

En el camino informó a sus compañeras lo que había hecho, y cuando llegó al colegio la llevaron a la enfermería. Pasado el mediodía, la Inspectora apenas se había dado cuenta de la situación, y lo primero que hizo fue acudir a la enfermería para instar a Paola a orar a Dios. También el Vicerrector y el médico del colegio se acercaron a la enfermería.

Según declaraciones de sus compañeras, ellas llamaron a su madre quien, como pudo, llegó al colegio en unos 30 minutos, y se llevó a su hija al Hospital en un taxi, donde le hicieron un lavado de estómago, pero Paola no mejoraba, por lo que la trasladaron a otra Clínica.

Un día después, el 13 de diciembre de 2002, por la mañana, Paola murió por intoxicación con fósforo blanco. La señora Petita Albarracín declaró que ese día, después de la muerte de su hija, el médico forense la llamó y le mostró el cuerpo de Paola desnudo y abierto, estando expuestos sus órganos. Conforme la declaración, el médico le enseñó una carnosidad pequeña y le dijo: "señora, éste es el útero de su hija, no hay embarazo".

Paola dejó tres cartas antes de morir. El texto de dos de ellas (una en borrador), iban dirigidas al Vicerrector, expresando que se sintió "engañada" por él, quien había "tenido" otras mujeres, por lo que decidió tomar veneno al no poder soportar "tantas cosas que sufría". 
3. Acciones de investigación, diligencias judiciales y administrativas posteriores a la muerte de Paola.

El 13 de diciembre de 2002 se llevó a cabo el levantamiento del cadáver en la morgue de la “Clínica Kennedy”. La autopsia concluyó que se trataba de un cadáver, sexo femenino, raza mestiza, 16 años de edad, 157 centímetros de estatura, muerte por edema agudo de pulmón.

El padre de Paola, Máximo Guzmán, denunció ante la Fiscalía la muerte de su hija, pidiendo que se investigara la responsabilidad del Vicerrector. Indicó que "es de dominio público que la decisión de ingerir el veneno con los diablillos se debió a una decepción amorosa, pues el Vicerrector del colegio, el señor Bolívar Eduardo Espín Zurita, había seducido a Paola", después pidió ampliar la investigación agregando que mediaron elementos de "intimidación, seducción, engaño, falsas promesas y violación”. A esta denuncia se adjuntaron cartas escritas por Paola, dirigidas a su madre y al señor Bolívar Espín. Sin embargo, cuando el Vicerrector fue llamado por la fiscalía rechazó la denuncia y acusaciones en su contra.

Por su parte, la madre de Paola, Petita Albarraicin, pidió tomarles declaración a las alumnas del colegio, pero las madres de éstas se negaron, porque las niñas ya habían sido amenazadas de ser expulsadas si declaraban ante la autoridad. Al final, solo hablaron dos compañeras.

En febrero del año 2003 se solicitó la detención del Vicerrector, sin embargo, al ir a su casa, se dieron cuenta de que ya se había fugado. Más tarde, Petita tuvo que recusar en dos ocasiones a los jueces por no resolver prontamente, y no fue hasta el año 2004, dos años después de la muerte de Paola, cuando ordenaron la localización y captura del vicerrector que, por supuesto, apeló la decisión a través de su abogado.

En 2005 la Corte Superior desestimó ese recurso presentado por el vicerrector, pero sí modificó el delito bajo el que le condenaban porque, a su parecer, los elementos del delito de acoso sexual no se cumplían en especie, debido a que el Vicerrector no persiguió a Paola, sino que ella "requirió sus favores docentes" para salir adelante en una materia. Él mismo se los ofreció a cambio de "relaciones sentimentales", siendo este "el principio de la seducción”. La Corte Superior consideró que la conducta del Vicerrector, si bien configuró estupro, definido por el Código Penal vigente al momento de los hechos como la cópula lograda empleando la seducción o el engaño, no hubo elementos de acoso sexual, pues Paola accedió voluntariamente, en palabras de la Corte Superior “requirió sus favores docentes".

En 2005, la Jueza que llevaba el caso suspendió el procedimiento hasta que pudieran encontrar al Vicerrector. Posteriormente, en el año 2008 se declaró caducada la acción penal a solicitud de la defensa y cesaron todas las medidas en contra del vicerrector; es decir, como no pudieron encontrarlo, decidieron archivar el caso.

La madre de Paola promovió dos juicios más para que el Vicerrector pagara al menos por el daño moral y gastos del juicio, pero su pretensión fue desestimada. Luego intentó demandar administrativamente al colegio por no proteger ni prestar asistencia a su hija, pero respondieron que no podía “confirmarse la supuesta relación amorosa”, y que no existía prueba de que el Vicerrector hubiera correspondido al "enamoramiento" de Paola. Lo único que hizo el colegio fue removerlo del puesto por un "presunto abandono injustificado del

№ 9, 2021. Página | 250 
cargo", pero no hizo señalamiento alguno de la violencia ejercida contra Paola y las otras estudiantes.

\section{Resolución de la Corte Interamericana de Derechos Humanos}

\section{El derecho de las niñas a una vida libre de violencia sexual en el ámbito educativo.}

La Corte comienza citando la Convención Belém do Pará, un instrumento regional que encuentra eco en el sistema universal a través de la Convención sobre la Eliminación de todas las formas de Discriminación contra la Mujer (CEDAW por sus sigas en inglés), así como en los sistemas regionales de protección de derechos humanos de las mujeres, tales como el Convenio de Estambúl en el espacio europeo, o el Protocolo de Maputo para el continente africano.

La Convención Belém do Pará ayuda a la Corte a precisar el concepto de violencia que utilizará en el examen de responsabilidad internacional para el Estado del Ecuador; así, la Corte señala que la violencia no puede limitarse al maltrato físico, sino que comprende cualquier acción o conducta basada en el género, que cause muerte, daño o sufrimiento físico, sexual o psicológico a las mujeres, tanto en el ámbito público como en el privado. Además el derecho de las mujeres a una vida libre de violencia, incluye también el derecho a no ser discriminada; por el contrario, las niñas y las mujeres deben ser valoradas y educadas libres de patrones estereotipados de comportamiento, así como de prácticas sociales y culturales basadas en conceptos de inferioridad o subordinación. La Corte continúa aludiendo a la misma Convención para destacar que en ella se contempla expresamente el acoso sexual en instituciones educativas como una forma de violencia contra las mujeres.

La Convención Belém do Pará indica además, que los países latinoamericanos deben abstenerse de realizar acciones o prácticas de violencia contra la mujer, así como vigilar que los funcionarios estatales tampoco lo hagan. Más aún, deben actuar con debida diligencia para prevenir, investigar y sancionar cualquier violación a los derechos humanos de las niñas y las mujeres, adoptando normas o medidas necesarias, inclusive administrativas, para posibilitar la prevención y sanción de dichas violaciones, así como procurar la erradicación de la violencia.

Tratándose este caso de una niña, la Corte menciona que la Convención sobre los Derechos del Niño manda a los Estados a adoptar medidas para proteger al niño contra toda forma de perjuicio o abuso físico o mental, descuido o trato negligente, malos tratos o explotación, incluido el abuso sexual, mientras el niño se encuentre bajo la custodia de los padres, de un representante legal o de cualquier otra persona que lo tenga a su cargo, incluidas, por supuesto, autoridades escolares. La Corte señaló que una educación que se imparte vulnerando derechos humanos es violatoria del derecho mismo a la educación, por eso es preciso que los Estados tengan en consideración la gravedad y las especificidades que presentan la violencia sexual, de género y contra la mujer; porque las niñas y niños tienen derecho a un entorno educativo seguro y a una educación libre de violencia sexual. En este sentido, debe tenerse en cuenta que las personas adolescentes, y las niñas en particular, suelen tener más probabilidades de sufrir actos de violencia, coacción y discriminación; por este motivo deben establecerse acciones para monitorear la problemática de la violencia sexual

No 9, 2021. Página | 251 
en instituciones educativas y desarrollar políticas para su prevención, facilitando mecanismos simples, accesibles y seguros para que los hechos puedan ser denunciados, investigados y sancionados.

\section{La violencia sexual sufrida por Paola}

De acuerdo a los hechos del caso, Paola entre los 14 y 16 años de edad mantuvo una relación sexual con el Vicerrector de su colegio.

La violencia sexual contra la mujer puede presentar diversos grados, de acuerdo a las circunstancias del caso y diversos factores, entre los que pueden encontrarse las características de los actos cometidos, su reiteración o continuidad y la vinculación personal preexistente entre la mujer y su agresor, o la subordinación de ella a éste, a partir de una relación de poder. También pueden resultar relevantes, de acuerdo al caso, condiciones personales de la víctima, como ser una niña.

En las circunstancias del caso, se produjo el abuso de una relación de poder y confianza, porque precisamente la persona que tenía el deber de cuidado en el ámbito escolar, es quien ejerce la violencia, poniendo a Paola en un estado de vulnerabilidad, el mismo que permitió la consumación de actos de violencia sexual contra ella.

a. El aprovechamiento de una relación de poder y la situación de vulnerabilidad: El vicerrector no era solamente una persona con autoridad escolar sino que, además, era un funcionario público, ese hombre con 40 años más que Paola tenía un rol de poder y deber de cuidado. La relación sexual, por lo tanto, se dio en el marco de una vinculación manifiestamente desigual; el Vicerrector, como autoridad académica, gozaba de una situación de superioridad frente a una niña estudiante.

Así, la vinculación sexual fue obtenida por el aprovechamiento de esa relación de poder y confianza, y comenzó precisamente como condición para que él la ayudara a pasar el año escolar. En este marco, los estereotipos de género tendientes a culpabilizar a la víctima, facilitaron el ejercicio del poder y el aprovechamiento de la relación de confianza, para naturalizar actos indebidos y contrarios a los derechos de Paola.

Durante una de las audiencias, la perita Casas señaló que la promesa de pasar de año funciona en la lógica del "grooming”, es decir, una cierta preparación para asegurar las condiciones del acercamiento afectivo y efectivo, en que el funcionario público, docente, posee una clara superioridad no solo por el cargo que detenta sino también por su edad. Paola es su blanco, identificada como tal no sólo por su vulnerabilidad de ser niña y adolescente, sino por su condición específica de alguien que presenta un mal rendimiento. Las promesas pueden constituirse en chantajes, "tu me das, yo te doy", en un marco de acoso sexual por chantaje.

La perita Ximena Cortés Castillo manifestó que la adolescente pertenecía además a una comunidad educativa vulnerable, por las condiciones sociales y del colegio, el mismo que toleró los actos del Vicerrector y que victimizaron a Paola. La Corte advirtió que el caso de Paola no fue un hecho aislado, pues habían existido otros casos similares en el colegio, conocidos por su personal directivo, pero nadie movió un dedo para denunciar o abordar la

No 9, 2021. Página | 252 
situación. Por el contrario, se ocultó lo que sucedía e incluso se culpabilizó y estigmatizó a Paola, señalándola como provocadora del vínculo con el Vicerrector y, una vez muerta, en vez de hacer justicia, se buscó procurar la impunidad. En ese sentido, una compañera de colegio de Paola señaló que fueron presionadas por el Presidente de la Asociación de Profesores para apoyar al Vicerrector.

De modo adicional, es relevante destacar que la educación recibida por Paola y sus compañeras no contemplaba conceptos relativos a su salud reproductiva, derecho a la autonomía y consentimiento informado. Al respecto, el perito Muñoz Villalobos ha resaltado la importancia de la educación sexual, y ha indicado que, de conformidad con los estándares internacionales vigentes, puede entenderse como un derecho humano en sí mismo y un medio imprescindible para fortalecer la educación en general. Agregó que órganos de la Organización de las Naciones Unidas han reconocido el derecho humano a la educación sexual integral, y han considerado que debe ser un componente obligatorio de la escolarización. En ese sentido, el derecho a la educación sexual y reproductiva integra el derecho a la educación y, como ha señalado el Comité DESC, entraña un derecho a una educación sobre la sexualidad y la reproducción que sea integral, que no sea discriminatoria, que esté basada en pruebas, que sea científicamente rigurosa y que sea adecuada en función de la edad. Dicha educación debe ser apta para posibilitar a las niñas y los niños un adecuado entendimiento de las implicaciones de las relaciones sexuales y afectivas, particularmente en relación con el consentimiento para tales vínculos y el ejercicio de sus derechos.

Paola no contó con educación que le permitiera comprender la violencia sexual implicada en los actos que sufrió, ni con un sistema institucional que le brindara apoyo para su tratamiento o denuncia. Por el contrario, la violencia referida fue convalidada, normalizada y tolerada por la institución.

b. El carácter discriminatorio de la violencia sufrida: La violencia sufrida, además, conllevó una forma de discriminación. La violencia sexual contra niñas no sólo expresa una discriminación prohibida en razón del género, sino que también puede resultar discriminatoria en función de la edad. Este es el caso de niñas y niños, quienes pueden verse afectados en forma desproporcionada y particularmente grave por actos de discriminación y violencia de género. La Corte ha señalado que el impacto de la violencia sexual en las niñas, niños y adolescentes víctimas, puede verse severamente agravado, por lo que podrían sufrir un trauma emocional diferenciado de los adultos, y un impacto sumamente profundo, en particular cuando el agresor mantiene un vínculo de confianza y autoridad con la víctima.

En virtud de la obligación de no discriminar, los Estados están obligados a adoptar medidas positivas para revertir o cambiar situaciones discriminatorias, existentes en sus sociedades, que vayan en perjuicio de determinado grupo de personas. Por eso, los Estados deben invertir en medidas proactivas que promuevan el empoderamiento de las niñas e impugnen las normas y los estereotipos patriarcales y otras normas y estereotipos de género perjudiciales, así como en reformas jurídicas para hacer frente a la discriminación directa e indirecta contra las niñas. Pese a ello, no consta que antes de diciembre de 2002 el Estado adoptara políticas que tuvieran un impacto efectivo en el ámbito educativo de Paola y que procuraran prevenir o revertir situaciones de violencia de género contra niñas en el marco de la enseñanza. Por lo expuesto, los actos de acoso y abuso sexual cometidos contra Paola no solo constituyeron,

No 9, 2021. Página | 253 
en sí mismos, actos de violencia y discriminación en que confluyeron, de modo interseccional, distintos factores de vulnerabilidad y riesgo de discriminación, como la edad y la condición de mujer. Esos actos de violencia y discriminación se enmarcaron, además, en una situación estructural, en la que pese a ser la violencia sexual en el ámbito educativo un problema existente y conocido, el Estado no había adoptado medidas efectivas para revertirlo.

\section{Responsabilidad del Estado por la violación al derecho a la vida de Paola}

La Corte ha señalado que el derecho a la vida abarca el derecho a una vida digna; es decir, no solo comprende el derecho de todo ser humano a no ser privado de la vida arbitrariamente, sino también el derecho a que no se le impida el acceso a las condiciones que le garanticen una existencia digna.

Los efectos de la violencia contra niñas o niños pueden resultar sumamente graves. La violencia contra niños o niñas tiene múltiples consecuencias, entre ellas, consecuencias psicológicas y emocionales (como sensaciones de rechazo y abandono, trastornos afectivos, trauma, temores, ansiedad, inseguridad y destrucción de la autoestima), que pueden derivar incluso en suicidio o intentos de cometerlo. En el presente caso, es claro que el Estado no solo no adoptó acciones para proteger a Paola, sino que directamente irrespetó sus derechos, no solo por los actos directos de violencia sexual, sino también por la tolerancia al respecto por parte de la institución educativa a la que asistía.

Paola, siendo niña y estando en una situación de vulnerabilidad particular, fue sometida durante un período superior a un año a una situación continuada de abuso y violencia institucional de carácter discriminatorio. Resulta claro que la violencia sexual generó un grave sufrimiento a Paola que, como ya se indicó, se hizo evidente a partir de su suicidio. Este acto se cometió el mismo día en que la madre de la adolescente estaba citada para concurrir al colegio. La conducta suicida mostró hasta qué punto el sufrimiento psicológico resultó severo. Las agresiones directas a los derechos de la niña y la tolerancia institucional respecto a las mismas generaron evidentes consecuencias perjudiciales en ella. La situación de violencia indicada implicó, entonces, una afectación al derecho de Paola a una existencia digna, que se vio estrechamente ligada al acto suicida que ella cometió.

Por otra parte, luego de que las autoridades estatales escolares tomaron conocimiento del riesgo concreto a la vida de Paola, por la ingesta de veneno, la conducta del Estado no fue diligente para procurar salvar su vida.

Las autoridades escolares no actuaron con la celeridad requerida. Paola fue llevada a la enfermería, dónde no consta que recibiera tratamiento alguno y la Inspectora General del colegio instó a Paola a pedir perdón a Dios. Fueron las compañeras de Paola quienes llamaron a la madre, quien logró llegar un tiempo después, cercano a 30 minutos, y llevó a su hija a un hospital y luego a la Clínica Kennedy, donde falleció al día siguiente.

La conducta estatal mencionada no resultó diligente. Esta conclusión es independiente de la carencia de atención médica en el propio colegio. Como ha indicado el Estado, no puede asimilarse el "deber de cuidado" exigido a un colegio en materia de salud al que es posible

No 9, 2021. Página | 254 
esperar en una institución hospitalaria. No obstante, las autoridades del colegio no trasladaron en forma inmediata a Paola a una institución que pudiera darle atención, omitiendo cumplir el deber de auxiliar a una persona sobre la cual tenían obligación de garantizar sus derechos. No sólo durante cerca de 30 minutos Paola estuvo sin atención o tratamiento alguno, sino que no se realizaron acciones para procurarlo, pese a estar bajo la custodia estatal y estando las autoridades al tanto de que la integridad física y la vida de la niña corrían riesgo. En el caso, es esto último, y no la falta de tratamiento médico en el colegio, lo que genera la responsabilidad estatal .

La Corte concluye que el Estado no respetó el derecho de Paola a una vida digna y no garantizó su vida al tomar conocimiento del riesgo de muerte, finalmente consumado, a partir de un acto suicida.

\section{Conclusión de la Corte}

La violencia sexual ejercida contra Paola, siendo ella una niña, afectó su derecho a una vida libre de violencia, resultó discriminatoria y menoscabó su posibilidad de decidir en forma autónoma su relacionamiento con otras personas y el ejercicio de su sexualidad. Vulneró también su derecho a la educación, que, incluye la observancia de los derechos humanos en el marco del proceso educativo. Asimismo, le causó graves sufrimientos y tuvo relación con su decisión de quitarse la vida. El Estado, además, no le prestó el auxilio debido para procurar evitar su muerte.

Por lo anterior, Paola vio lesionados sus derechos a la vida, a la integridad personal, a la vida privada y a la educación. El Estado incumplió su deber de respetar los derechos señalados, mediante el ejercicio de violencia sexual contra Paola, y también su deber de garantizarlos. Ecuador incumplió su obligación de proveer medidas de protección a Paola en su condición de niña, como también de abstenerse de cualquier acción o práctica de violencia contra la mujer y velar por que las autoridades, sus funcionarios, personal y agentes e instituciones se comporten de conformidad con esta obligación. Tampoco actuó con la diligencia debida para prevenir esa violencia ni adoptó las medidas necesarias a tal efecto. El incumplimiento del Estado de sus obligaciones de respeto y garantía implicó la inobservancia de su deber de cumplir las mismas sin discriminación.

\section{Reflexión final. La necesidad de educar en igualdad real}

El caso de Paola, es uno de muchos que pudo salir del anonimato gracias a la fuerza incansable de Petita, una madre destrozada que convirtió su dolor en lucha y perseverancia. A pesar de que el caso de Paola es el primero que llega a la Corte Interamericana de Derechos Humanos, respecto al tema de violencia sexual en el ámbito educativo, las brechas de desigualdad siguen siendo profundas para las mujeres y niñas.

Paola murió siendo incapaz de reconocer la violencia sexual de la que era víctima; su familia no supo de esta situación durante más de un año; el colegio naturalizaba la violencia sexual y encubría a los violadores amenazando a las alumnas; los tribunales ecuatorianos dictaron sentencias plagadas de estereotipos, que concebían la posibilidad de que una niña con 40 años menos que su agresor, fuera una seductora en busca de favores sexuales. Lo que queda

No 9, 2021. Página | 255 
claro en este caso, es que todo lo anterior, se materializó gracias a un mecanismo que trastoca todo el sistema social y cultural, el mismo que genera un pacto de silencio y encubrimiento que permite, tolera y es cómplice de la injusticia.

Mientras no tengamos claro que la educación no es solamente un derecho humano fundamental, sino un elemento clave para el desarrollo sostenible, seguirá habiendo muchas niñas como Paola, que serán infravaloradas por quienes detentan el poder, niñas convertidas en objetos sexuales de mentes pervertidas. Por si esto fuera poco, el escenario de pandemia acentúa las desigualdades y violaciones a los derechos humanos de niñas como Paola, que todos los días deben luchar en campos de batalla, con frecuencia sus propios hogares, para poder sobrevivir.

Burzynska y Contreras (2020) señalan dos motivos principales por los que es más factible que las niñas no regresen a los centros educativos después de la pandemia: un mayor riesgo de que sean sometidas a explotación sexual, se queden embarazadas o contraigan matrimonio (forzoso) durante los confinamientos; y un aumento desproporcionado del trabajo doméstico no remunerado, que provoca que las niñas dediquen más tiempo a ayudar en el hogar que a estudiar. Este es el panorama actual, y si no se toman medidas inmediatas, la pandemia machista, capaz de resistir a cualquier pandemia de salud, seguirá cobrando vidas y arrebatando infancias. Es momento de educar en igualdad real hoy, desde los espacios más cercanos, en cada trinchera donde tengamos agencia, por mínima o insignificante que parezca, porque ningún esfuerzo es pequeño cuando se trata de tener esperanza en que un mundo mejor es posible.

\section{Referencias}

Unicef (2018). Niños y niñas en América Latina y el Caribe. Panorama 2018. Recuperado de https://t.ly/cqPa

Ocde (2018). OECD Handbook for Internationally Comparative Education Statistics 2018: Concepts, Standards, Definitions and Classifications, OECD Publishing, Paris. Recuperado de https://t.ly/tV1q

World Bank (2018). Missed opportunities: The high cost of not educating girls. Recuperado de https://t.ly/6K5b

Unicef, onu mujeres, únete latinoamérica. (2014). La prevención de la violencia contra las mujeres y las niñas el contexto educativo Prácticas promisorias en 14 países de América Latina y El Caribe. Documento de trabajo. Recuperado de https://t.ly/nvEj

Contreras, J. M.; Bott, S.; Guedes, A.; Dartnall, E. (2010). Violencia sexual en Latinoamérica y el Caribe: análisis de datos secundarios. Iniciativa de Investigación sobre la Violencia Sexual. Recuperado de https://t.ly/Eri3

Corte Interamericana de Derechos Humanos. Caso Guzmán Albarracín y otras Vs. Ecuador. Fondo, Reparaciones y Costas. Sentencia de 24 de junio de 2020. Serie C No. 405. 
Burzynska K. y Contreras, G. (2020), Gendered effects of school closures during the COVID19 pandemic, The Lancet, Vol. 395(10242) p. 1968. 